\title{
Precise Optical Spectroscopy with Ion Traps*
}

\author{
Wayne M. Itano, J. C. Bergquist, Randall G. Hulet, and D. J. Wineland \\ Time and Frequency Division, National Bureau of Standards, Boulder, Colorado 80303, U.S.A.
}

Received September 24, 1987; accepted October 8, 1987

\begin{abstract}
We have used stored ion methods to improve resolution and sensitivity in optical spectroscopy. Single atomic ions have been confined by electric and magnetic fields, cooled by laser radiation pressure to temperatures on the order of $1 \mathrm{mK}$, and probed spectroscopically with narrowband lasers. The absorption resonance of a single $\mathrm{Hg}^{+}$ion has been observed by a decrease in the transmitted light intensity. An ultraviolet transition in $\mathrm{Hg}^{+}$has been observed with a linewidth of only $30 \mathrm{kHz}$. Quantum jumps to and from metastable levels of $\mathrm{Hg}^{+}$have been observed and used to determine radiative decay rates and to infer the existence of photon antibunching. Quantum jumps have also been observed in single $\mathrm{Mg}^{+}$ions.
\end{abstract}

\section{Introduction}

Ion traps have only recently been used in obtaining high resolution optical spectra, although their advantages have been realized for a long time. Microwave spectra of trapped ions, on the other hand, have been observed with high resolution since the 1960s $[1,2]$. Elsewhere in these Proceedings, G. Werth discusses some recent experiments in this area [3]. A few years ago, at the Ninth International Conference on Atomic Physics, the field of optical spectroscopy of trapped ions was reviewed [4]. At that time, the highest resolution that had been achieved in the optical domain corresponded to a linewidth of about $3 \mathrm{MHz}$ at an effective wavelength of $2.1 \mu \mathrm{m}$ [5]. In a recent experiment to be discussed later in this Paper, an ultraviolet transition was observed with a fractional resolution about three orders of magnitude better than this [6].

Some general problems which tend to limit the resolution with which optical spectra are observed are Doppler shifts, perturbations due to collisions or to electric and magnetic fields, and the limited observation time. At least in a few favorable cases, these problems can be greatly alleviated for ions stored in traps, so that we are left with the natural linewidth. For a transition with a stable lower level, the natural linewidth (in hertz) is the inverse of the mean radiative lifetime (in seconds) of the upper level, divided by $2 \pi$.

The Doppler shift is usually divided into a term which is linear in the atomic velocity, called the first-order Doppler shift, and a part which is quadratic, called the second-order Doppler shift. The high intensity and narrow frequency width of laser light sources make it possible to apply various nonlinear spectroscopic techniques, such as saturated absorption or multiphoton absorption, that cancel the effects of firstorder Doppler shifts [7]. However, the second-order Doppler shift, which is a result of relativistic time dilation, still remains. For typical laboratory conditions, this shift is only about one part in $10^{12}$, but in some cases, it may seriously

\footnotetext{
* Contribution of the National Bureau of Standards. Not subject to copyright in the U.S.
}

limit the resolution or accuracy of the measurement. One example is the work of Barger et al. on the $657 \mathrm{~nm}$ transition of calcium, which has a frequency of $4.57 \times 10^{14} \mathrm{~Hz}$ and a natural linewidth of $410 \mathrm{~Hz}$ [8]. Resonances with linewidths as small as $2 \mathrm{kHz}$ were observed, but they were severely shifted and distorted by the second-order Doppler shift.

If both the first- and second-order Doppler shifts are to be reduced, the atoms must be cooled. An atomic vapor placed in a conventional refrigeration device would quickly condense on the walls of the container. But laser cooling, a method by which laser radiation pressure is used to reduce the velocities of atoms, achieves the cooling without contact with material objects [9]. The technique was proposed independently by Hänsch and Schawlow for free atoms [10] and by Wineland and Dehmelt for trapped ions [11]. Laser cooling to temperatures on the order of $1 \mathrm{mK}$ has been demonstrated with some kinds of atoms and atomic ions. This reduces the second-order Doppler shifts to the level where they are presently not a problem. An atom or atomic ion of mass $100 \mathrm{u}$ (unified atomic mass units) cooled to $1 \mathrm{mK}$ has a second-order Doppler shift of 1.4 parts in $10^{18}$.

The observed resonance linewidth cannot be much less than the inverse of the observation time. In the case of the work on calcium, the observation time was limited to about $0.3 \mathrm{~ms}$ by the time it took the atoms to pass through the $21 \mathrm{~cm}$ long resonance region [8]. In some experiments, the observation times have been increased by confining the atoms with specially coated walls or by buffer gases, but collisions with the wall or buffer gas molecules shifted and broadened the observed resonances. Ions can be trapped for long periods by electric and magnetic fields. External field perturbations of carefully chosen transition frequencies can be less than a part in $10^{15}[12,13]$. Collisions are almost entirely absent in the ultra-high-vacuum environment of an ion trap. Neutral atoms have been trapped by static magnetic fields [14-16] and by optical fields [17], but these trapped atoms have not yet been studied spectroscopically. In these traps, the fields responsible for trapping would also have perturbed the resonances.

\section{Sensitive detection of ions}

A single atomic ion can easily be observed by the laserinduced fluorescence of an allowed transition, once it has been cooled so that the Doppler broadening of the transition is small. In this case, a single ion may scatter as many as $10^{8}$ photons per second. Even if only a small fraction of the photons, around $10^{-4}$ in typical experimental cases, are collected and counted, the fluorescence can easily be observed [18].

The apparatus used at the National Bureau of Standards 


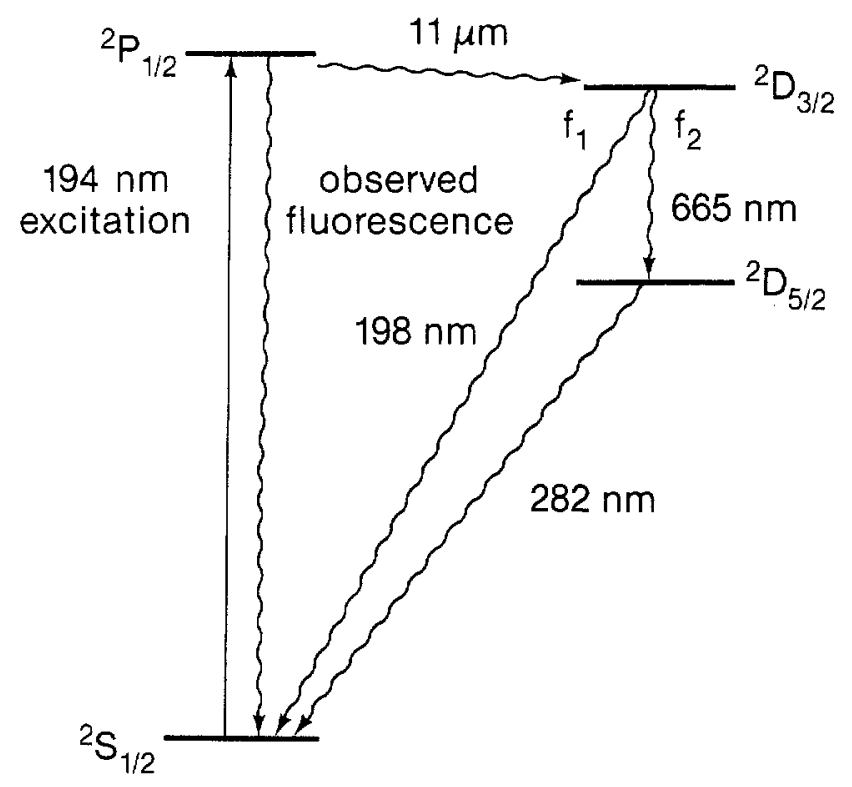

Fig, 1 . The lowest energy levels of $\mathrm{Hg}^{+}$and the transition wavelengths. The decay rates from all three excited levels were determined by observing only the $194 \mathrm{~nm}$ fluorescence. (From Ref. [33]).

for experiments with $\mathrm{Hg}^{+}$has been described previously $[19,20]$. The $\mathrm{Hg}^{+}$ions were confined in a radiofrequency (Paul) trap under ultra-high-vacuum conditions. In one case, a single $\mathrm{Hg}^{+}$ion has been kept in the trap for over a week. The lowest energy levels of $\mathrm{Hg}^{+}$are shown in Fig. 1. The $5 d^{10} 6 s^{2} S_{1 / 2}$ ground level is connected to the $5 d^{10} 6 p^{2} P_{1 ; 2}$ level by a strong electric dipole transition. The $5 d^{9} 6 s^{22} D_{3 / 2}$ and the $5 d^{9} 6 s^{2}{ }^{2} D_{5 / 2}$ levels are metastable. In order to laser cool and optically detect the ions, a few microwatts of c.w. $194 \mathrm{~nm}$ radiation were required. This radiation was generated by sum-frequency mixing the output of a frequency doubled $514.5 \mathrm{~nm} \mathrm{Ar}^{+}$laser with the output of a $792 \mathrm{~nm}$ dye laser in a potassium pentaborate crystal [21]. The $194 \mathrm{~nm}$ fluorescence was collected by a lens system and detected by a photomultiplier tube. As many as 60000 photons per second were detected from a single ion.

\subsection{Single-ion absorption detection}

In a recent experiment, an optical transition in a single $\mathrm{Hg}^{+}$ ion was detected by observing the decrease in the intensity

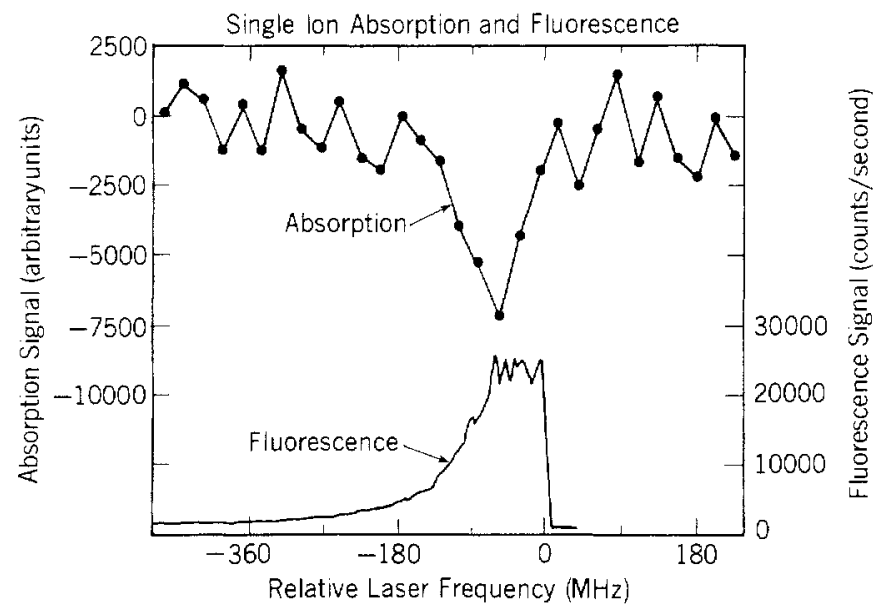

Fig. 2. The $194 \mathrm{~nm}$ resonance of a single $\mathrm{Hg}^{+}$ion due to absorption (upper trace) and fluorescence (lower trace), detected at the same time. (From Ref. [22]). of the $194 \mathrm{~nm}$ beam transmitted through the trap [22]. Radiofrequency modulation techniques were used in order to avoid low frequency noise, such as that due to amplitude fluctuations of the $194 \mathrm{~nm}$ radiation. A maximum of about $10^{-5}$ of the light was absorbed. Figure 2 shows the absorption signal and the fluorescence signal, taken at the same time. The modulation and phase-sensitive detection of the absorption resulted in a signal which was approximately proportional to the frequency derivative of the fluorescence signal. Laser heating caused the fluorescence signal to drop suddenly to zero when the frequency was tuned above resonance. The integration time per point on the absorption curve was $50 \mathrm{~s}$.

\subsection{Electron shelving}

Detecting a narrow (weakly allowed) transition by observing fluorescence from the same transition would be very difficult. A double-resonance method originally proposed by Dehmelt [23] makes it possible to detect such transitions with very high efficiency. The method can be applied to $\mathrm{Hg}^{+}$(see Fig. 1). The narrow, weakly-allowed, $282 \mathrm{~nm}$ transition is detected as follows: The ion is assumed to be initially in the ground level. Light at a wavelength near $282 \mathrm{~nm}$ is pulsed on, possibly driving the ion to the ${ }^{2} D_{5 / 2}$ level. Light at a wavelength near $194 \mathrm{~nm}$ is then pulsed on. If the ion had made a transition in the previous step, no fluorescence would be observed; otherwise an easily detectable fluorescence signal would be observed. The method is called "electron shelving", since the optically active electron is temporarily shelved in the upper level of the weak transition.

\section{Quantum jumps}

The electron shelving techniques suggests a method of observing quantum jumps. If light near both resonance wavelengths is present simultaneously, then the fluorescence observed from the strong transition should turn off and on abruptly as the atom makes transitions to and from the metastable level. The dynamics of this process was investigated theoretically by Cook and Kimble [24] and later by others [25]. Quantum jumps of single ions were observed by this method in $\mathrm{Ba}^{+}[26,27]$, in $\mathrm{Hg}^{+}[20]$, and recently in $\mathrm{Mg}^{+}$[28].

Quantum jumps have also been observed by us in $\mathrm{Hg}^{+}$ with only the $194 \mathrm{~nm}$ radiation present. Once excited to the ${ }^{2} P_{1 / 2}$ level, the ion usually decays back to the ground level, but it has a probability of about $10^{-7}$ to decay to the ${ }^{2} D_{3 / 2}$ level. The probability is small because the transition frequency is low and because it requires configuration mixing to occur (the nominal configurations differ in two orbitals). From the ${ }^{2} D_{3 / 2}$ level, the atom decays either directly to the ground level with probability $f_{1}$ or to the ${ }^{2} D_{5 / 2}$ level with probability $f_{2}=1-f_{1}$. The $194 \mathrm{~nm}$ fluorescence jumps between a steady level (the "on" state) when the ion is cycling between the ground level and the " $P_{1 / 2}$ level and zero (the "off" state) when it is in either the ${ }^{2} D_{3: 2}$ level or the ${ }^{2} D_{5 / 2}$ level.

Typical fluorescence data are shown in Fig. 3 for one, two, and three ions. The fluorescence intensity jumped from one discrete level to another and was proportional to the number of ions in the "on" state. For these data, only the $194 \mathrm{~nm}$ radiation was present. The intensity was high enough that quantum jumps due to the weak ${ }^{2} P_{1 / 2}$ level to ${ }^{2} D_{3 / 2}$ level decay occurred several times per second. 


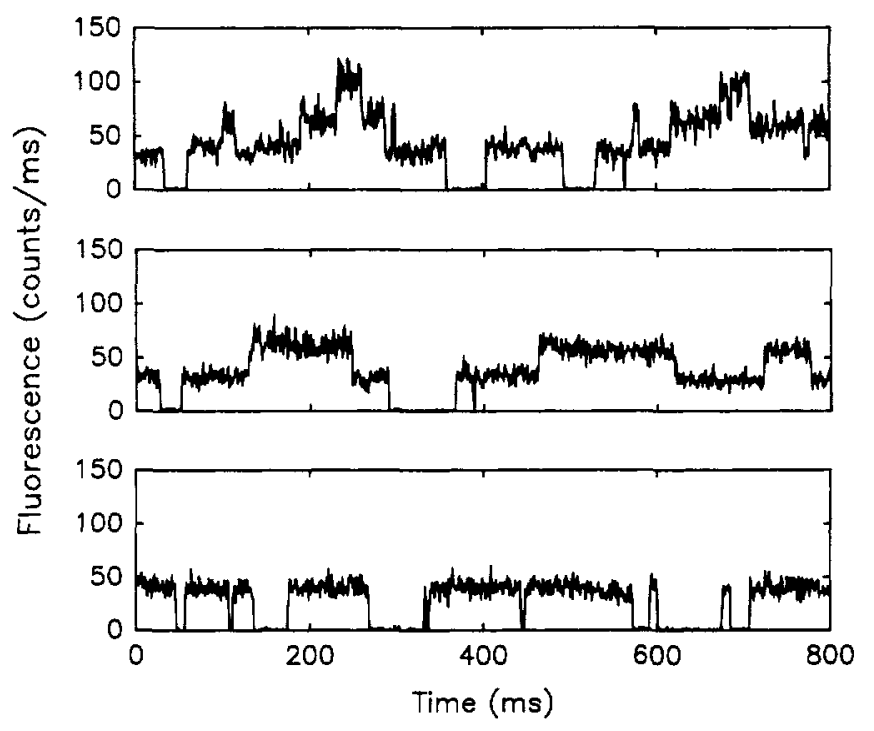

Fig. 3. Fluorescence intensities as a function of time for three $\mathrm{Hg}^{+}$ions (top trace), two $\mathrm{Hg}^{+}$ions (middle trace), and one $\mathrm{Hg}^{+}$ion (lowest trace). The fluorescence takes a step down when an ion makes a transition to the metastable ${ }^{2} D_{3 / 2}$ level and takes a step up when an ion returns to the ground level from a metastable level. The integration time per point is $1 \mathrm{~ms}$ and the points are connected by straight lines. (From Ref. [33]).

\subsection{Quantum jumps of two and three ions}

Sauter et al. have reported observing multiple quantum jumps, that is, simultaneous quantum jumps of two or more ions, when several $\mathrm{Ba}^{+}$ions were stored in the same trap [29]. They attributed this phenomenon to a cooperative interaction between the atoms and the radiation field. We have examined our data to test the statistical independence of the quantum jumps of two or more simultaneously trapped ions.

One test was based on measuring the fractions of the time that the ions spent in each of the possible fluorescence levels. The probability distribution of fluorescence intensities was plotted for the data from one, two, and three ions. The distribution for one ion is shown in Fig. 4. The counts at low intensity (left side of plot) are due to stray scattered light when the ion is in the "off" state; the peak at high intensity (right side) is due to the fluorescence of the ion in the "on" state. The solid curve is a least-squares fit of the data to a sum of a Poisson distribution (for the left peak) and a Gaussian

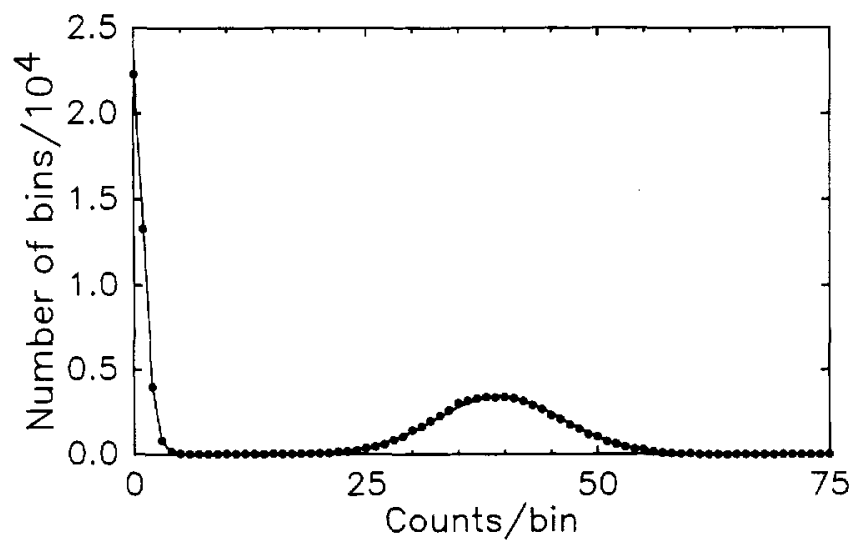

Fig. 4. Fluorescence intensity distribution for one $\mathrm{Hg}^{+}$ion. The numbers of $1 \mathrm{~ms}$ time bins in which a given number of photons was detected are plotted as dots. The solid curve is a least-squares fit to the data.

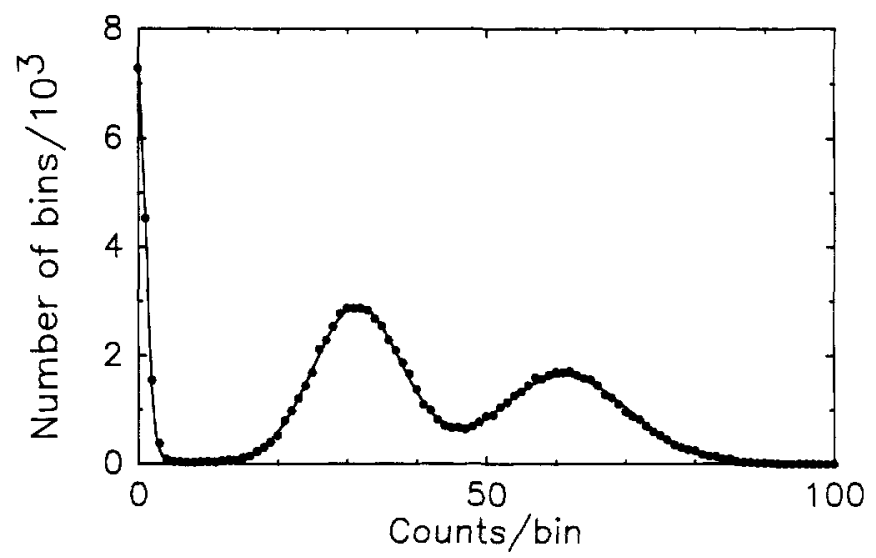

Fig. 5. Fluorescence intensity distribution for two $\mathrm{Hg}^{+}$ions (dots) and a least-squares fit (solid curve).

distribution (for the right peak). The standard deviation (sigma) of the fitted Gaussian is about $8 \%$ larger than the square root of the mean, which is the expected value due to statistical fluctuations. This is probably due to intensity and frequency fluctuations of the $194 \mathrm{~nm}$ source. The ratio of the area under the Poisson distribution to that under the Gaussian distribution is a measurement of the ratio of the time that the ion was in the "off" state to the time that it was in the "on" state $(0.71$ for this data set). Plots of the two-ion and three-ion intensity distributions and least-squares fits are shown in Figs. 5 and 6 . The areas under the peaks of the distributions are proportional to the amounts of time that the fluorescence was at each of the various discrete levels. The curves fitted the data well, even though the peaks were not completely resolved.

Let $p_{\text {off }}$ and $p_{\text {on }}$ be the probabilities that a single ion is in the "off" state or the "on" state, respectively. Consider two ions subjected to the same $194 \mathrm{~nm}$ intensity and acting independently of each other. In this case, the probability $p_{0}$ that both are in the "off" state is equal to $\left(p_{\text {off }}\right)^{2}$; the probability $p_{1}$ that one ion is in the "off" state and the other in in the "on" state is equal to $2 p_{\text {on }} p_{\text {off }}=2 p_{\text {on }}\left(1-p_{\text {on }}\right)$; and the probability $p_{2}$ that both are in the "on" state is equal to $\left(p_{\text {on }}\right)^{2}$. Similar relationships hold for three ions. The leastsquares fit to the two-ion data yielded the values $p_{0}=0.139$, $p_{1}=0.472$, and $p_{2}=0.389$. If $p_{1}$ is (arbitrarily) chosen to calculate the value of $p_{\text {on }}$, then the prediction, based on the assumption of independence, is that $p_{0}=0.145$ and

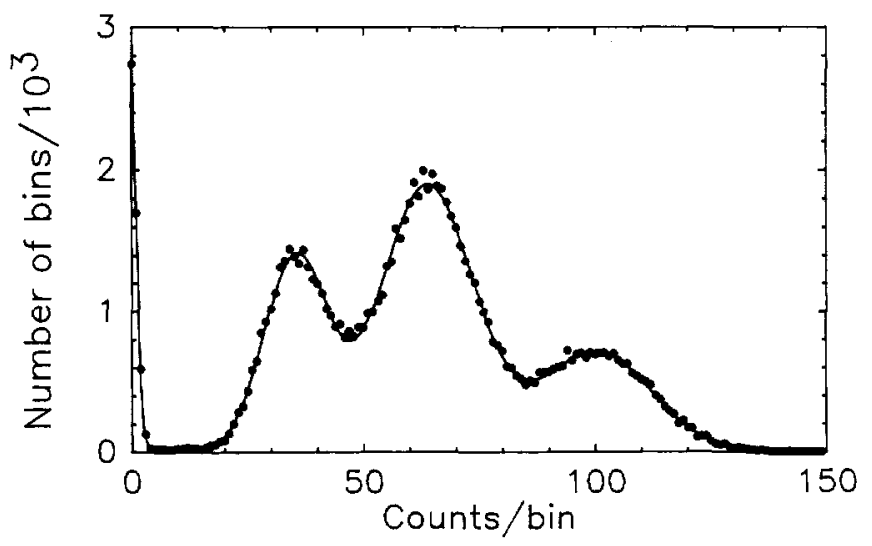

Fig. 6. Fluorescence intensity distribution for three $\mathrm{Hg}^{+}$ions (dots) and a least-squares fit (solid curve). 


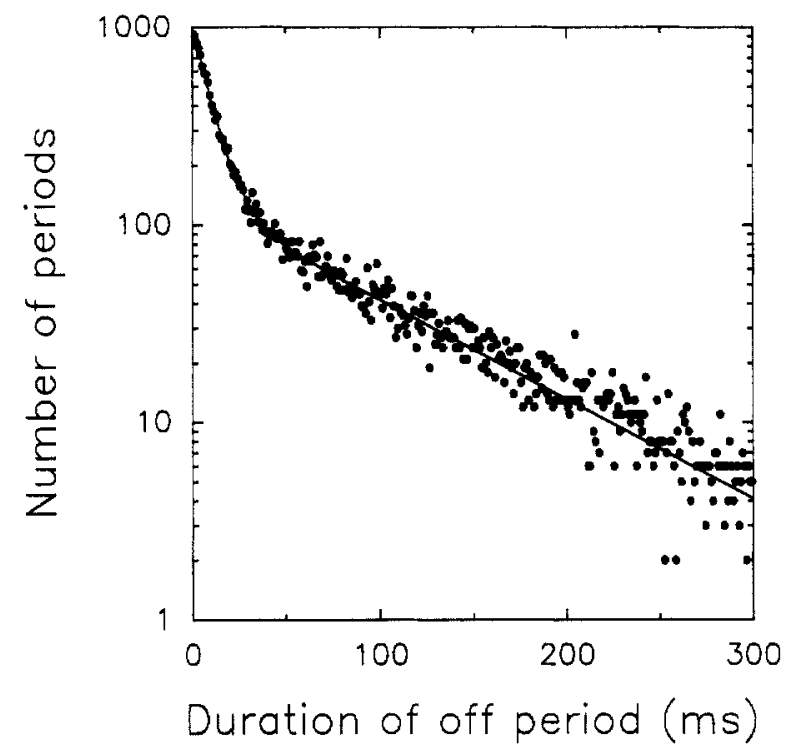

Fig. 7. Distribution of fluorescence-off periods for a single $\mathrm{Hg}^{+}$ion as a function of the duration of the periods (dots) and a least-squares fit. (From Ref. [33]).

$p_{2}=0.383$. For the three-ion data, let $p_{n}(n=0,1,2,3)$ be the probability that $n$ ions are in the "on" state. The leastsquares fit yielded the values $p_{0}=0.053, p_{1}=0.244, p_{2}=$ 0.485 , and $p_{3}=0.218$. If $p_{3}$ is used to calculate the value of $p_{\text {on }}$, the prediction based on independence is that $p_{0}=0.061$, $p_{1}=0.286$, and $p_{2}=0.433$. Thus, the two- and three-ion data agreed fairly well with the assumption of independence of the ions. The discrepancies from the predictions were most likely due to effects of intensity and frequency variations of the $194 \mathrm{~nm}$ radiation source, which are not included in the simple model used to fit the data.

In another test of independence, a search through the two-ion data was made for double quantum jumps. Over 500 consecutive jumps, which took place in a period of $20 \mathrm{~s}$, were tabulated. A total of five apparent double quantum jumps were found, which is approximately the number that would be expected due to random coincidences within the finite resolving time of the instrumentation (1 ms).

\subsection{Measurements of radiative decay rates}

The radiative decay rates of the ${ }^{2} D_{3 / 2}$ level and the ${ }^{2} D_{5 / 2}$ level are reflected in the probability distribution $W_{\text {off }}$ of the durations of the "off" periods for a single ion irradiated by $194 \mathrm{~nm}$ radiation. A calculation based on the rate equations for the probabilities of being in the various levels yields

$W_{\text {off }}(\tau) \propto\left[f_{2} \gamma_{2} \exp \left(-\gamma_{2} \tau\right)+\left(f_{1} \gamma_{1}-\gamma_{2}\right) \exp \left(-\gamma_{1} \tau\right)\right]$.

Here $\gamma_{1}$ and $\gamma_{2}$ are the total radiative decay rates of the ${ }^{2} D_{3 / 2}$ level and the ${ }^{2} D_{5 ; 2}$ level respectively.

The experimental fluorescence-off distribution was leastsquares fitted to eq. (1) to obtain values for $\gamma_{1}, \gamma_{2}$, and $f_{1}$. Figure 7 shows the data and the least-squares fit. The values obtained from the fit are $\gamma_{1}=109 \pm 5 \mathrm{~s}^{-1}, \gamma_{2}=11.6 \pm$ $0.4 \mathrm{~s}^{-1}$, and $f_{1}=0.491 \pm 0.015$. These values are in fair agreement with calculations $[30,31]$. The value of $\gamma_{2}$ is in good agreement with previous measurements $[19,20,32]$, but the value of $\gamma_{1}$ is about a factor of 2 higher than the only previously reported value [32]. No previous measurements of $f_{1}$ exist. The measurements will be described in more detail elsewhere [33].

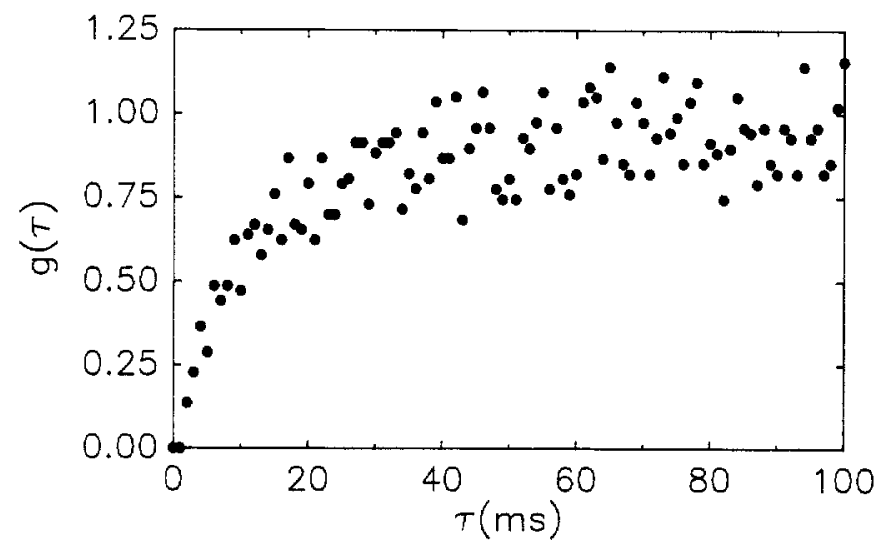

Fig. 8. Intensity correlation function of the $11 \mu \mathrm{m}$ radiation field generated by the radiative decay from the ${ }^{2} P_{1 / 2}$ level to the ${ }^{2} D_{3 / 2}$ level in a single $\mathrm{Hg}^{+}$ ion, showing the photon antibunching. The data from several runs were averaged.

\subsection{Photon antibunching}

The two-time intensity correlation function of a light field is defined by $C(\tau)=\langle I(t) I(t+\tau)\rangle$, where $I$ is the intensity. A field is said to exhibit photon antibunching if $C(0)<C(\infty)$. No classical field can have this property, so a quantum theory is required to explain it. The quantum jumps of a single $\mathrm{Hg}^{+}$ ion from the "on" state to the "off" state, as in the lowest trace of Fig. 3, are assumed to mark the emission of an $11 \mu \mathrm{m}$ photon (see Fig. 1), even though these photons have not been observed directly. The probability density $g(\tau)$ that the emission of an $11 \mu \mathrm{m}$ photon is followed a time $\tau$ later by the emission of another one is proportional to $C(\tau)$ for the $11 \mu \mathrm{m}$ field. We expect $g(\tau)$ to go to zero for small $\tau$, since the ion has to decay to the ground level and be excited to the ${ }^{2} P_{122}$ level again before it can emit another $11 \mu \mathrm{m}$ photon. The function $g(\tau)$ (normalized to 1 as $\tau \rightarrow \infty$ ) was calculated for several one-ion runs and averaged to obtain the graph shown in Fig. 8, which clearly shows photon antibunching. A similar graph, showing photon antibunching in the $282 \mathrm{~nm}$ radiation emitted when light at both $194 \mathrm{~nm}$ and $282 \mathrm{~nm}$ was applied, has been shown previously [20].

\subsection{Quantum jumps in $\mathrm{Mg}^{+}$}

Quantum jumps of a single ${ }^{24} \mathrm{Mg}^{+}$ion in a Penning trap were investigated recently [28]. A single $280 \mathrm{~nm}$ radiation source was present and was tuned close to the $m_{J}=-1 / 2$ to $m_{J}=-3 / 2$ Zeeman component of the transition from the $3 s$ ${ }^{2} S_{1 / 2}$ ground level to the $3 p^{2} P_{3 / 2}$ level. The fluorescence intensity dropped to zero when the ion was driven to the $m_{J}=$ $+1 / 2$ sublevel of the ground level by a spontaneous Raman transition, and returned to its previous level when another spontaneous Raman transition drove the ion back to the $m_{J}=-1 / 2$ sublevel. A trace of the fluorescence intensity as a function of time is shown in Fig. 9. The data are in agreement with the theoretical prediction that the ratio of the time when the fluorescence is on to the time when it is off is very close to 16 [34]. This ratio is nearly independent of the $280 \mathrm{~nm}$ intensity because of the existence of coherences between excited levels. The experimental results provide a high precision $(2 \%)$ verification of an effective two-state rate equation description of the dynamics of the quantum jumps. 


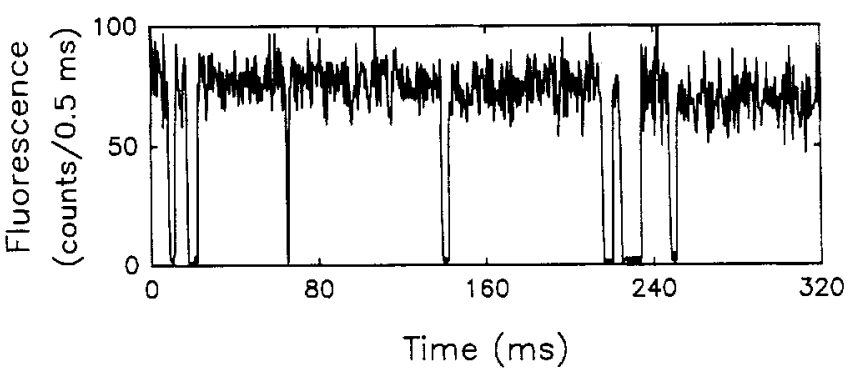

Fig. 9. Fluorescence intensity of a single $\mathrm{Mg}^{+}$ion as a function of time, showing the sudden changes due to quantum jumps to and from the groundstate Zeeman level which is not in resonance with the incident radiation. (Adapted from Ref. [28])

\section{Suppression of Doppler broadening by confinement}

In 1953 Dicke showed that the confinement of an atom to a region smaller than the resonance-radiation wavelength leads to a suppression of the first-order Doppler shift broadening [35]. This condition is the basis for the observation of narrow Mössbauer transitions of the nuclei in solids. It is also easy to satisfy this condition, commonly called the Lamb-Dicke criterion, for a microwave resonance, since the wavelengths can be several centimeters long. Microwave frequency standards such as the atomic hydrogen maser make use of this fact. In the optical domain, it can be satisfied for a single, laser-cooled ion confined in a trap. The residual first-order Doppler broadening takes the form of discrete resonances, displaced from the unshifted central resonance (the carrier), by multiples and combinations of the frequencies of motion of the ion. These extra resonances are called motional sidebands.

\subsection{High resolution spectra of $\mathrm{Hg}^{+}$}

We have used the shelved-electron method to detect the weak ${ }^{2} S_{1 / 2}$ to ${ }^{2} D_{5 / 2}$ transition of a single $\mathrm{Hg}^{+}$ion [6]. If the fluorescence was high enough to indicate that the ion was cycling between the ${ }^{2} \mathrm{~S}_{1 / 2}$ and the ${ }^{2} P_{1 / 2}$ levels, the $194 \mathrm{~nm}$ radiation was turned off and the $282 \mathrm{~nm}$ radiation was turned on for $20 \mathrm{~ms}$,

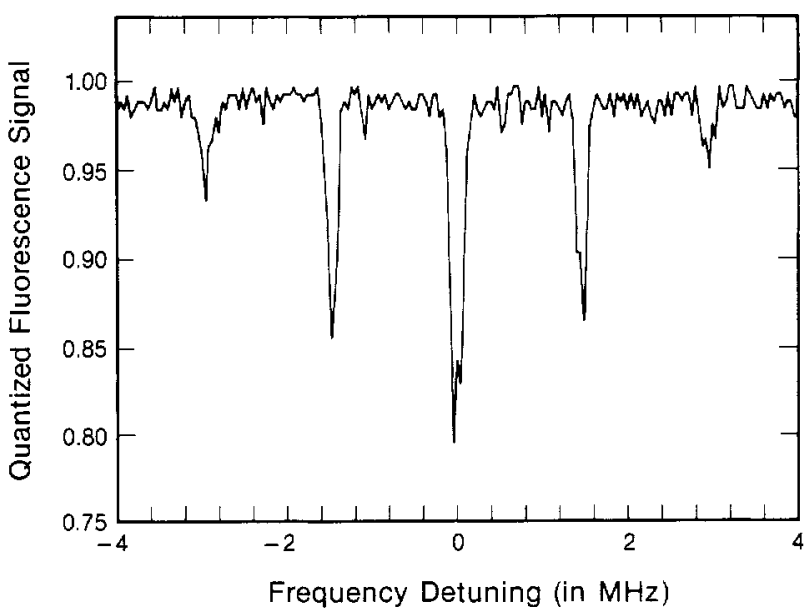

Fig. 10. The ${ }^{2} S_{1,2}$ to ${ }^{2} D_{5 / 2}$ resonance due to absorption of $282 \mathrm{~nm}$ radiation from a frequency-doubled dye laser by a single $\mathrm{Hg}^{+}$ion. The shelved electron method was used to detect this weakly allowed transition. The weaker absorption lines (motional sidebands) are separated from the central resonance by multiples of the frequencies of harmonic motion of the trapped ion and are due to the Doppler shift of the $282 \mathrm{~nm}$ radiation in the frame of the ion. (From Ref. [6]).

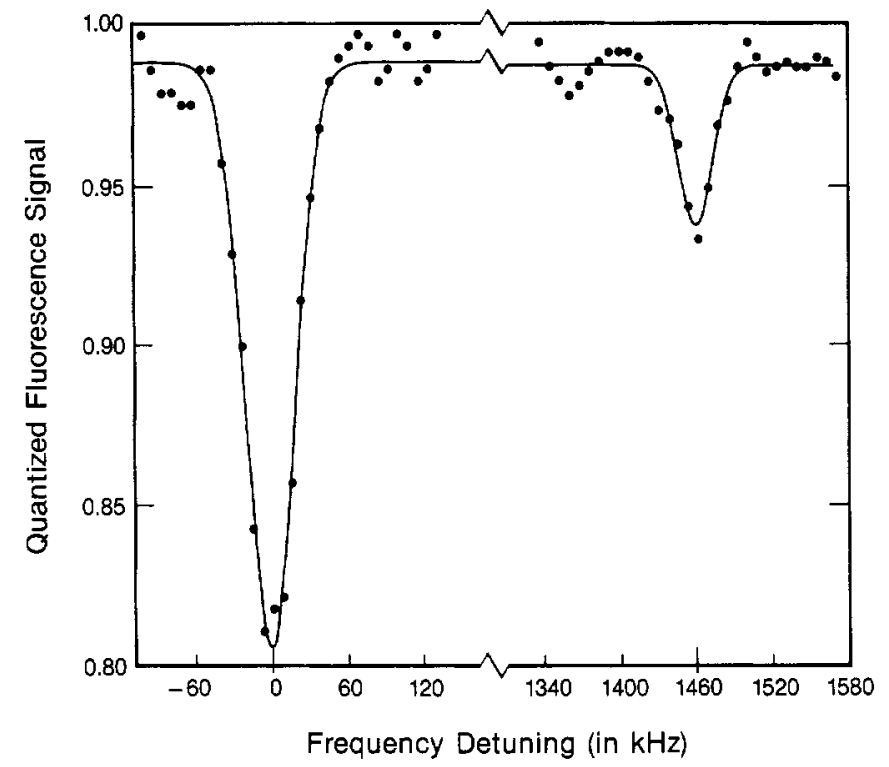

Fig. 11. High resolution frequency scan of the ${ }^{2} S_{1 / 2}$ to ${ }^{2} D_{5,2}$ resonance, showing the central resonance and the first upper motional sideband. From Ref. [6]).

which is much less than the ${ }^{2} D_{5 / 2}$ level lifetime. The $194 \mathrm{~nm}$ radiation was turned on again, and the fluorescence photons were counted for $10 \mathrm{~ms}$ to see whether the ion had made a transition to the ${ }^{2} D_{5 / 2}$ level. The result of the measurement was a 0 or a 1 , depending on whether the number of photons detected was below or above a threshold level. This eliminated certain kinds of instrumental noise, such as intensity fluctuations of the detection laser, leaving only the inherent quantum fluctuations of the atom. Figure 10 shows a spectrum taken in the manner just described, displaying the carrier and two motional sidebands on each side. Figure 11 shows a high resolution scan of the carrier and the first upper sideband. The resonances are approximately $30 \mathrm{kHz}$ wide. The ratio of intensities of the carrier and sideband indicates that the ion was cooled to near the theoretical minimum of $1.7 \mathrm{mK}$. The limit of $30 \mathrm{kHz}$ resolution was due to laser frequency fluctuations. If a suitably stabilized laser were used, the resolution would approach the natural linewidth of about $2 \mathrm{~Hz}$. The sensitivity of the transition frequency to fluctuations in the magnetic field could essentially be eliminated by using a suitable Zeeman component in either ${ }^{199} \mathrm{Hg}^{+}$or ${ }^{201} \mathrm{Hg}^{+}[36]$.

\section{Acknowledgements}

We gratefully acknowledge the support of the Air Force Office of Scientific Research and the Office of Naval Research.

\section{References}

1. Dehmelt, H., Adv. At. Mol. Phys. 3, 53 (1967); ibid, 5, 109 (1969)

2. Wineland, D. J., Itano, W. M. and Van Dyck, Jr., R. S., Adv. At, Mol. Phys. 19, 135 (1983).

3. Werth, G., these Proceedings.

4. Wineland, D. J., Itano, W. M., Bergquist, J. C., Bollinger, J. J. and Prestage, J. D., Atomic Physics 9 (Edited by R. S. Van Dyck, Jr. and E. N. Fortson), p. 3. World Scientific, Singapore, 1985

5. Janik, G., Nagourney, W. and Dehmelt, H., J. Opt. Soc. Am. B 2, 125 ! (1985).

6. Bergquist, J. C., Itano, W. M. and Wineland, D. J., Phys. Rev. A 36, 428 (1987). 
7. High-Resolution Laser Spectroscopy (Edited by K. Shimoda). Springer Verlag, Berlin, 1976; Levenson, M. D., Introduction to Nonlinear Laser Spectroscopy. Academic Press, New York, 1982.

8. Barger, R. L., Bergquist, J. C., English, T. C. and Glaze, D. J., Appl. Phys, Lett. 34, 850 (1979); Barger, R. L., Opt. Lett. 6, 145 (1981).

9. Wineland, D. J. and Itano, W. M., Phys. Today 40, 34 (June 1987),

10. Hänsch, T. W. and Schawlow, A. L., Opt. Commun. 13, 68 (1975).

11. Wineland, D. J. and Dehmelt, H., Bull. Am. Phys. Soc. 20, 637 (1975).

12. Dehmelt, H. G., IEEE Trans. Instrum. Meas. IM-31, 83 (1982).

13. Wineland, D. J., Science 226, 395 (1984).

14. Migdall, A. L., Prodan, J. V., Phillips, W. D., Bergeman, T. H. and Metcalf, H. J., Phys. Rev. Lett. 54, 2596 (1985).

15. Bagnato, V. S., Lafyatis, G. P., Martin, A. G., Raab, E. L., AhmadBitar, R. N. and Pritchard, D. E., Phys. Rev. Lett. 58, 2194 (1987).

16. Hess, H. F., Kochanski, G. P., Doyle, J. M., Masuhara, N., Kleppner, D. and Greytak, T. J., Phys. Rev, Lett. 59, 672 (1987).

17. Chu, S., Bjorkholm, J. E., Ashkin, A., and Cable, A., Phys. Rev. Lett. 57, 314 (1986).

18. Neuhauser, W., Hohenstatt, M., Toschek, P. E. and Dehmelt, H., Phys. Rev. A22, 1137 (1980)

19. Bergquist, J. C., Wineland, D. J., Hemmati, H., Daniel, H.-U. and Leuchs, G., Phys. Rev. Lett. 55, 1567 (1985).

20. Bergquist, J. C., Hulet, R. G., Itano, W. M. and Wineland, D. J., Phys. Rev. Lett. 57, 1699 (1986).

21. Hemmati, H., Bergquist, J. C. and Itano, W. M., Opt. Lett. 8, 73 (1983).

22. Wineland, D. J., Itano, W. M. and Bergquist, J. C., Opt. Lett. 12, 389 (1987).

23. Dehmelt, H. G., Bull. Am. Phys. Soc. 20, 60 (1975).
24. Cook, R. J. and Kimble, H. J., Phys. Rev. Lett. 54, 1023 (1985).

25. Javanainen, J., Phys. Rev. A33, 2121 (1986); Schenzle, A., DeVoe, R. G. and Brewer, R. G., Phys. Rev. A33, 2127 (1986); CohenTannoudji, C. and Dalibard, J., Europhys. Lett. 1, 441 (1986); Pegg, D. T., Loudon, R. and Knight, P. L., Phys. Rev. A33, 4085 (1986); Schenzle, A. and Brewer, R. G., Phys. Rev. A34, 3127 (1986); Kimble, H. J., Cook, R. J., and Wells, A. L., Phys. Rev. A34, 3190 (1986); Zoller, P., Marte, M. and Walls, D. F., Phys. Rev. A35, 198 (1987); Nienhuis, G., Phys. Rev. A35, 4639 (1987); Porrati, M. and Putterman, S., Phys. Rev. A36, 929 (1987).

26. Nagourney, W., Sandberg, J. and Dehmelt, H., Phys. Rev. Lett. 56, 2797 (1986).

27. Sauter, Th., Neuhauser, W., Blatt, R. and Toschek, P. E., Phys. Rev. Lett. 57, 1696 (1986).

28. Hulet, R. G., Wineland, D. J., Bergquist, J. C., and Itano, W. M., (submitted for publication)

29. Sauter, Th., Neuhauser, W., Blatt, R. and Toschek, P. E., Opt. Commun. 60, 287 (1986).

30. Garstang, R. H., J. Res. Natl. Bur. Stand. Sect. A68, 61 (1964).

31. Al-Salameh, D. and Silfvast, W., AT\&T Bell Laboratories, Holmdel, NJ, unpublished calculations.

32. Johnson, C. E., Bull. Am. Phys. Soc. 31, 957 (1986).

33. Itano, W. M., Bergquist, J. C., Hulet, R. G, and Wineland, D. J,, Phys. Rev, Lett. (in press).

34. Hulet, R. G. and Wineland, D. J., Phys. Rev. A 36, 2758 (1987).

35. Dicke, R. H., Phys. Rev. 89, 472 (1953).

36. Wineland, D. J., Itano, W. M., Bergquist, J. C. and Walls, F. L., in Proc. 35th Annual Symposium on Frequency Control, p. 602. Electronic Industries Association, Washington, D.C., 1981. 